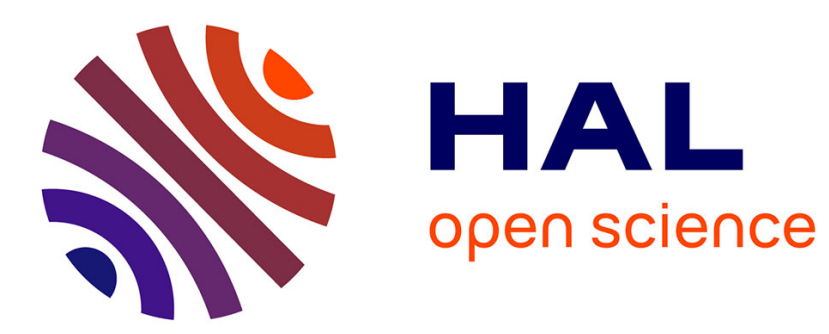

\title{
Effects of the Acknowledgment Traffic on the Capacity of Wireless Mesh Networks
}

Christelle Molle, Marie-Emilie Voge

\section{To cite this version:}

Christelle Molle, Marie-Emilie Voge. Effects of the Acknowledgment Traffic on the Capacity of Wireless Mesh Networks. 69th IEEE Vehicular Technology Conference (VTC2009-Spring), Apr 2009, Barcelone, Spain. pp.1-5, 10.1109/VETECS.2009.5073522 . hal-00434057

\section{HAL Id: hal-00434057 https://hal.science/hal-00434057}

Submitted on 20 Nov 2009

HAL is a multi-disciplinary open access archive for the deposit and dissemination of scientific research documents, whether they are published or not. The documents may come from teaching and research institutions in France or abroad, or from public or private research centers.
L'archive ouverte pluridisciplinaire HAL, est destinée au dépôt et à la diffusion de documents scientifiques de niveau recherche, publiés ou non, émanant des établissements d'enseignement et de recherche français ou étrangers, des laboratoires publics ou privés. 


\section{Effects of the Acknowledgment Traffic on the Capacity of Wireless Mesh Networks}

\author{
Christelle Molle \\ MASCOTTE, INRIA-I3S(CNRS/UNSA) \\ Sophia-Antipolis, France \\ Email : christelle.molle@sophia.inria.fr
}

\author{
Marie-Emilie Voge \\ POPS, INRIA-CNRS-USTL \\ Villeneuve d'Ascq, France \\ Email : marie-emilie.voge@liff.fr
}

\begin{abstract}
Since the emergence of ubiquitous computing, evaluating wireless network performances has become one of the major economic issues. Among the existing performance indicators, the network capacity, defined as the maximal amount of flow carried by a topology during a fixed time period, is essential. Some cross-layer characteristics have to be taken into account in order to optimally allocate the common resources. In this article, a comparative study is done between interference consequences in the two following models: (i) usual IEEE 802.11 MAC layer with acknowledgments at each hop, and (ii) block acknowledgments reported at the transport layer that can be included in the IEEE 802.16 standard. Cross-layer properties are modeled in a linear programming formulation that is solved using the column generation process. We quantify the gain in capacity induced by the move of the MAC acknowledgments into the transport layer, and show the better load distribution obtained in the network with the second model.
\end{abstract}

\section{INTRODUCTION}

In the past few years, wireless mesh networks (WMNs) have emerged as a key technology to provide ubiquitous highspeed services. They are a cost-effective solution that offers good reliability, with a simpler infrastructure than cellular networks, and better coverage than hot spots [1]. In urban areas, they evolve into the next generation of ubiquitous networks, providing better services for a large variety of applications like video, sound, voice...Laying competitive WMNs is a necessary condition to the development of these new applications.

In WMNs, nodes are comprised of mesh routers and mesh clients. The routers automatically establish and maintain mesh connectivity among themselves, forming a fixed infrastructure that is dynamically self-organized and self-configured. Mobile mesh clients, moving on a potentially unlimited area, access services like Internet through special routers called mesh gateways as depicted in Figure 1.

A very important requirement for the network performance evaluation is to maximize its capacity, i.e. the throughput offered to each flow. Theoretical network capacity has been investigated by numerous studies [2], [3], [4], [5]. One of the major issue in wireless networking is the capacity reduction when the network size, i.e. the number of nodes, increases. This is mainly due to interferences arising between radio transmissions between neighboring nodes [6], [7]. Limiting these interferences is therefore necessary, either at the equipment level (using directional antennas, orthogonal codes or

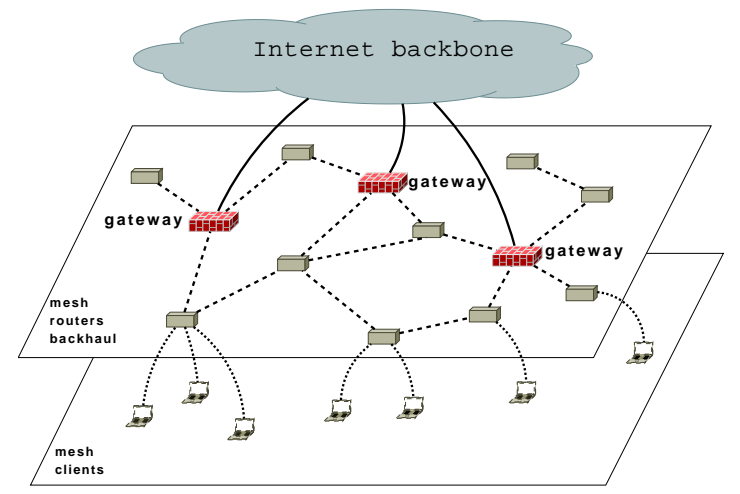

Fig. 1. Mesh routers and gateways form a fixed infrastructure providing Internet access to mobile mesh clients.

frequencies), or at the protocol level like in 802.11 when nodes listen to the radio channel or 802.16 in mesh mode in which a base station allows the channel access.

In this paper, we evaluate the performances of a periodic synchronous single-channel wireless mesh network in which communications are scheduled in order to avoid simultaneous contending transmissions. Our work is based on the round weighting problem introduced by [8] in which each set of compatible transmissions, i.e. a round, is assigned a weight corresponding to its activation duration during a time period. The objective is to minimize the sum of the round weights, i.e. the length of the time period. In a steady state of the network, the round weighting corresponds to a link schedule that allows routers-to-gateways routing establishment. Traffic requirements from every router can therefore be sent on these routes at maximum rate.

This work is based on a first formulation of the joint routing and scheduling problem for wireless mesh networks [9]. The authors considered a binary symmetric interference model in which acknowledgment traffic is integrated into the MAC layer like in the 802.11 Wifi standard with a channel access based on CSMA/CA. The main contribution of this paper is to measure and analyse the gain in capacity induced by another interference model modeling a different acknowledgment policy. The second model introduced corresponds to the move of the acknowledgments to the transport layer.

The rest of the paper is organized as follows. We first 
describe the network model in the next section. In Section IV, we present the linear formulation to model the cross-layer network characteristics and obtain the theoretical optimal capacity. Then in Section V, we analyse the results obtained by doing a comparative study that evaluates the effects of the acknowledgment traffic on the network capacity and load. We quantify this gain depending on the network topology.

\section{RELATED WORK}

Solutions to maximize the capacity of wireless multi-hop networks have been proposed in the literature. In [4], authors present a multi-channel scheduling over a routing tree for 802.16 based wireless mesh networks.

In [5], authors look at the complementary problem of finding a link schedule during a time period that minimizes the maximum delay induced by the re-emission of packets by the forwarding nodes.

In optimization problems for wireless mesh networks in the literature, the common interference model chosen is the binary distance two interference model presented in the next section, in which acknowledgments are modeled at each hop like in the 802.11 standard [6], [7], [9].

Our study is based on the problem formulation proposed and studied in [9] where the authors' objective was to maximize the minimum flow throughput that can be achieved by appropriately configuring the network in terms of the sets of links to activate, the flow routes, and the link activation schedule.

In this paper, we specifically quantify the effects of the move of the acknowledgments from the MAC layer to the transport layer on the wireless mesh network capacity. The traffic load in each router is also investigated in order to estimate the amount of flow each mesh router has to forward.

\section{NETWORK MODEL}

We consider the fixed infrastructure of a wireless mesh network forming a wireless backhaul (Fig. 1). This backhaul is comprised of mesh routers (MRs) interconnected by wireless links and integrated with Internet through special nodes called mesh gateways (MGs). We globally call mesh points (MPs) the union of MRs and MGs. Each MR has a traffic requirement corresponding to the aggregated traffic demand of the mesh clients connected to it. These requests have to be sent from the MRs to Internet, i.e. the MGs, through multi-hop paths.

Mesh points in the WMN all use omnidirectional antennas and have the same transmission power. We therefore model the network as a directed graph $G=(V, E)$, where $V$ is the set of MPs, and $E$ is the set of possible radio transmissions between any pair of nodes in the network based on the transmission range of each MP.

We study two different protocols for wireless networks that have the same technical and traffic settings but differ in their interference model.

\section{A. Technical model}

We consider periodic, scheduled WMNs in which all nodes have only one wireless interface that operates on the same frequency band (or channel).
In order to avoid contentions, communications are periodically scheduled. A time period is divided into slots of equal length and each slot is then assigned to a round, i.e. a set of pairwise non-interfering transmissions. A link can be selected in different rounds, and thus it can be activated during several slots of a period.

\section{B. Traffic model}

At each time period, a mesh router $v_{k}$ sends $d_{k}$ units of traffic in the network to Internet through the gateways. Given a link scheduling during one period, routing one unit of traffic up to the gateways can need several periods.

In the two different interference models described in the next subsection, acknowledgments are considered as traffic flow going on the link opposite to the data transmission. We set the acknowledgment traffic as a percentage $\alpha$ from the total data traffic of a link. Communication time alloted for this acknowledgment traffic in the network can be used either (i) at the MAC layer, or (ii) at the transport layer. One can note that the second model enforces a symmetric routing for the acknowledgment traffic in the transport layer.

\section{Interference models}

In both models, we first assume the half-duplex property: a node cannot send and receive traffic simultaneously. Moreover, we assume that each node has an interference range that is slightly greater than its transmission range. Therefore, two neighboring nodes cannot transmit simultaneously since they are within interference range of each other. Similarly, a node cannot receive a transmission if its neighbors are transmitting at the same time.

The difference between the two models resides in the traffic exchanges occurring between two nodes during a time slot of the period.

(i) Symmetric model: In the first case, acknowledgments are included into a one hop communication and are controlled at the MAC layer like in the 802.11 standard. A node sending traffic must then be able to receive the acknowledgment without interferences from other transmissions. Similarly, a node must acknowledge the received traffic without interfering with other receptions. Thus, during a time slot, a communicating node is both a sender and a receiver. This is translated into a symmetric distance-2 interference model as depicted into a grid topology in Figure 2.

(ii) Asymmetric model: When acknowledgments disappear from the MAC layer, a sending node does not need to be a receiver anymore: it does not wait for immediate acknowledgments at this level. Similarly, a receiving node is only a receiver. As depicted in Figure 3, two neighboring nodes can now send traffic simultaneously to two different receivers. Acknowledgments have now to be scheduled at the transport layer like traffic flow. We therefore call this model the asymmetric distance-1 interference model. 


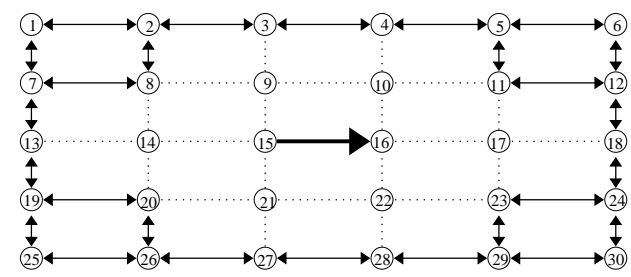

Fig. 2. Symmetric distance-2 interference model in a grid network. Activation of link $(15,16)$ blocks all communications in the 2-neighborhood of the link since node 16 sends an ACK to node 15 instantly.

\section{OPTIMIZATION OF THE NETWORK CAPACITY}

We use the following capacity measure: for a set of traffic requests, we compute the minimum time period length needed to route all the requests from the routers to the gateways through multi-hop paths. We allow a fractional multi-path routing from one mesh router to the gateways. Indeed, we would like to consider not only a router-to-gateway oriented traffic, but also return traffic as well as transverse traffic between two routers. Fractional routing allows, for a given bandwidth allocation, to route more traffic leading to a capacity increase in comparison to a more constrained mono-routing.

\section{A. Problem formulation}

Given the network graph $G=(V, E)$, a subset $\mathcal{A P} \subset V$ of MGs, and a set of requests $K$, the problem consists in finding a routing such that all the requests $d_{k} \in K$ from router $v_{k}$ to the gateways are satisfied in a minimum period of time. This means that we need to compute routes whose links can be activated during a time period of minimum length. This is equivalent of maximizing the flow rate given traffic requirements: if $\lambda$ units of flow are sent from a router to a gateway in $T$ units of time, then the flow rate is $\lambda / T$ in steady state.

In the following linear program, $f_{p} \in \mathbb{R}^{+}$is the flow variable over path $p \in P$, where $P$ is the set of all possible paths in the graph $G$. The integer variable $a_{r}$ corresponds to the number of time slots assigned to round $r \in R$, where $R \subseteq \mathcal{P}(E)$ with $\mathcal{P}(E)$ containing the set of transmissions in the network that are pairwise non-interfering. $\mathcal{P}_{k}$ is the set of paths from source node $v_{k}$.

$$
\begin{gathered}
\min \sum_{r \in R} a_{r} \\
\sum_{p \in \mathcal{P}_{k}} f_{p}=d_{k}, \quad \forall k \in K \\
\sum_{p \in P, p \ni e} f_{p}+\alpha \sum_{p \in P, p \ni x_{e}} f_{p} \leqslant \sum_{r \ni e} a_{r}, \quad \forall e \in E
\end{gathered}
$$

Objective (1) seeks to minimize the sum of the round activation durations. Routing the traffic requests in a minimum period of time is equivalent of maximizing the per-node flow rate as explained above. Constraints (2) are the demand satisfaction constraints: each MR $v_{k}$ has a traffic requirement $d_{k}$ that has to be routed to the set of MGs over a set of

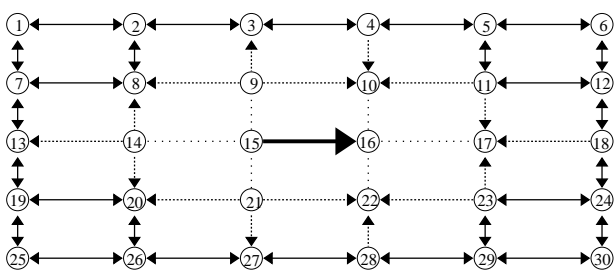

Fig. 3. Asymmetric distance-1 interference model in a grid network. Activation of link $(15,16)$ does not block reception of neighbors of 16 and transmission of neighbors of 15 since ACK $(16,15)$ is delayed.

paths contained in $\mathcal{P}_{k}$. Constraints (3) link the routing and the scheduling and can be compared to capacity constraints: flow carried by a link is limited by its time activation during the period. But, if a link $e$ carries some flow, then the acknowledgment traffic has to be carried by the link $x_{e}$ depending on the interference model considered. Actually, $x_{e}=e$ in the symmetric model since the activation of $e$ includes both the traffic and acknowledgment sending. In the asymmetric model, $x_{e}$ becomes $\bar{e}$, the opposite link of $e$, because we have to schedule the acknowledgments flow on $\bar{e}$ during the time period.

\section{B. Resolution method}

The model is then solved using the column generation process [10], that is a prominent technique to cope with largescale linear programs with an exponential set of variables. Its efficiency on routing problems has been shown in [9], [11], [12]. It is a primal-dual process that solves the continuous relaxation of our problem that corresponds to the Round Weighting Problem [8].

The sets $P$ and $R$ of possible paths and rounds have an exponential size. Column generation allows to limit the set of generated variables needed for the computation of the optimal solution. More precisely, the process runs as follows:

(i) We start with restricted sets of paths and rounds allowing a feasible solution of the problem: $R_{O}=\{\{e\}, \forall e \in$ $E\}$, and $P_{0}=\{$ shortest paths from each router $v \in V \backslash$ $\mathcal{A P}$ to a gateway $\in \mathcal{A P}\}$.

(ii) We then solve program $(1)-(3)$ and obtain primal and dual optimal solutions for this current set of variables.

(iii) Given the dual reduced costs, we seek for new paths and rounds violating the following constraints of the dual program:

$$
\begin{aligned}
\alpha \sum_{e \in p} y_{e} & \geqslant x_{k}, \forall k \in K, p \in \mathcal{P}_{k} \\
1 & \geqslant \sum_{e \in r} y_{e}, \forall r \in \mathcal{R}
\end{aligned}
$$

We compute the new variables using auxiliary linear programs.

(iv) We add these paths and rounds to the current set of variables and repeat (ii)-(iii). The separation theorem claims that solving again the program $(1)-(3)$ produces a better solution [13]. 


\begin{tabular}{|c|c|c|c|c|}
\hline \multirow{2}{*}{ Topology } & \multicolumn{2}{|c|}{ Symmetric model } & \multicolumn{2}{c|}{ Asymmetric model } \\
\cline { 2 - 5 } & Mean round size & \# paths & Mean round size & \# paths \\
\hline 3x3 grid - center & 1.33 & 8 & 2.03 & 8 \\
\hline $3 \times 3$ grid - corners & 1.57 & 6 & 3 & 9 \\
\hline $7 \times 7$ grid - corners & 9.3 & 61 & 13.96 & 73 \\
\hline 10x10 grid - 10 gtws & 19.04 & 158 & 27.78 & 173 \\
\hline 10 nodes - 1 gtw & 1 & 9 & 1.43 & 12 \\
\hline 50 nodes - 1 gtw & 4.69 & 55 & 6.74 & 61 \\
\hline 100 nodes - 2 gtws & 7.76 & 106 & 11.5 & 116 \\
\hline
\end{tabular}

TABLE I

THE MEAN SIZE OF THE ROUNDS AND THE NUMBER OF PATHS IN THE OPTIMAL SOLUTIONS ARE INCREASED WITH THE ASYMMETRIC MODEL.

(v) When no such paths or rounds exist, then duality theory claims that both the primal and the dual are optimized.

One can notice that special QoS requirements can be easily added in the column generation process, like limiting the number of hops in the paths, or getting more fairness by selecting specific links in the rounds.

\section{Simulations}

The column generation process has been implemented in Java programming language using the Mascopt graph library developed by team members [14]. Linear programs have been solved using Ilog Cplex solver.

Tests have been realized on regular grid topologies (Manhattan-like topologies), as well as on random graphs in which a fixed number of nodes has been deployed on a rectangular plane following a Poisson law. Then, a fixed transmission power constructs the transmission area of each node. If a node is within the transmission range of another node, then a link is constructed between them.

The percentage $\alpha$ of capacity reserved for the acknowledgment traffic varies from 1 to $10 \%$. Traffic requests have been generated either uniformly with value 1 , or randomly with values between 1 and 20 for each router. Several gateway placements have been computed, either regular for grids (in the center and/or the corners), or random in more general topologies.

\section{A. Gain in capacity}

The asymmetric interference model constructs rounds containing more edges (the mean size increases by 50\%) since a transmission will not block all its 2-neighborhood (see Table I). Therefore the capacity obtained with this model is greater than the one with the symmetric model.

As depicted in figure 4(a), the gain in capacity for grid topologies is always greater than $20 \%$, and can be close to $50 \%$ depending on gateway distribution and network size. One can notice that, when the grid has one gateway placed in the center, the ratio of the capacities from the two models is constant with the acknowledgment size $(\alpha)$. Similarly, the variation is slow with 4 gateways situated at the corners of the grid. This phenomenon comes from the bottleneck region located around each gateway that restricts the optimal solution of our problem [9]. A regular gateway placement also allows a fair distribution of the traffic flow between the gateways. In

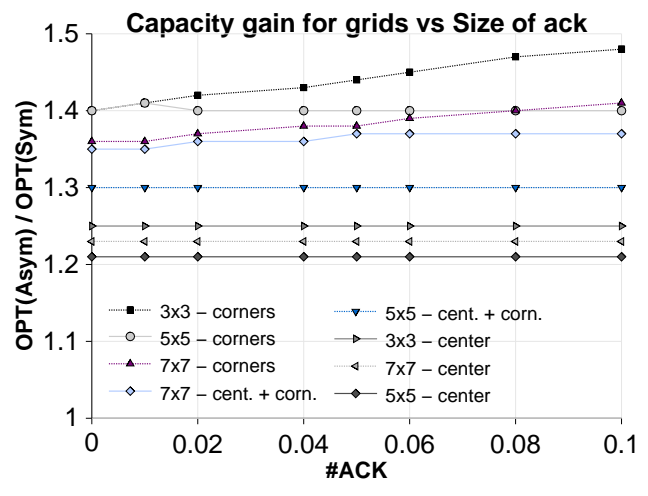

(a) Grid topologies.

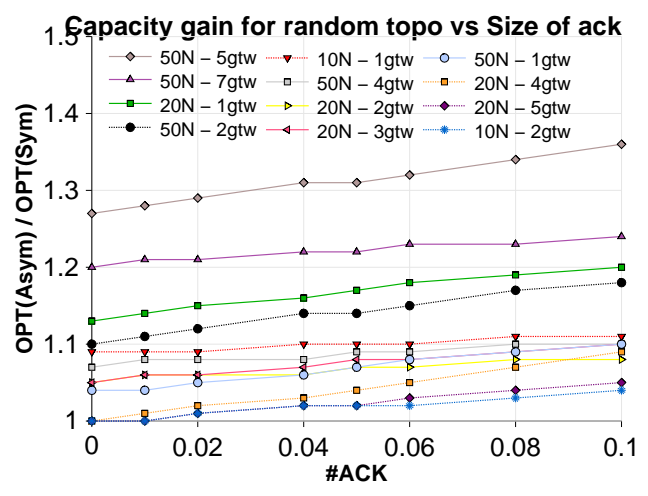

(b) Random topologies.

Fig. 4. Capacity gain induced by the move of acknowledgments from the MAC layer to the transport layer.

these regular topologies with special connectivity properties, links are activated enough to schedule the acknowledgment traffic in the reverse links.

In random networks, this phenomenon disappears. As depicted in Figure 4(b), the gain in capacity is growing with $\alpha$, the size of the the acknowledgment traffic. It stays lower than the gain in the grid topologies, i.e. between 0 and $20 \%$, since the node distribution and the gateway placement is completely random.

\section{B. Network load distribution}

We also investigated the network load within the two models. We call load of a mesh router the amount of flow received 


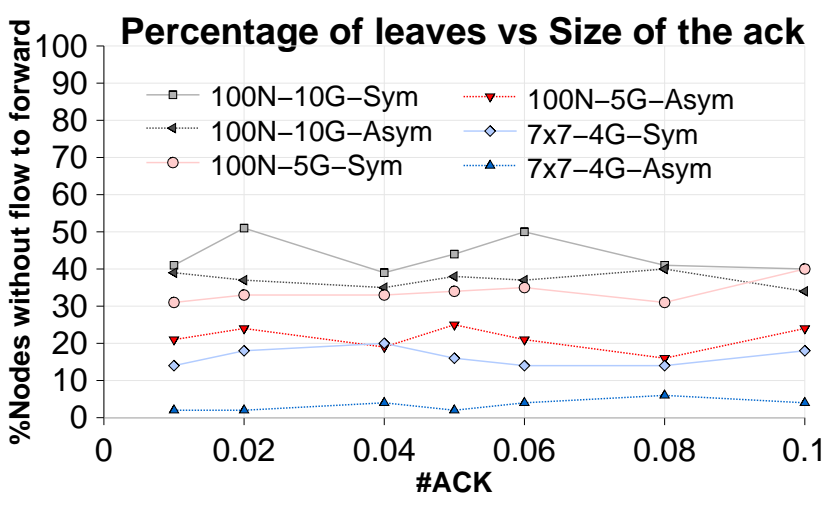

Fig. 5. The network load is more spread among the routers in the asymmetric model since there is less nodes without flow traffic to forward.

from other routers that it has to forward. Simulations point out that the asymmetric model gives a better load distribution.

The mean load is really close between the two models, but we have remarked that there is a more important variation between the nodes with the symmetric model. As for an example, Figures 6(a) and 6(b) shows the load distribution into a random network of 50 nodes. One can remark that there is more nodes with an important amount of data to forward in the symmetric model ( 6 nodes have more than 10 units of flow to forward whereas in the asymmetric model they are only 3 ).

Figure 5 depicts the percentage of leaves in the network. We call leaf a mesh router that sends only its flow traffic, without forwarding any other flow. Following the definition, leaves have load 0 . One can see that, for each topology, the curve with the asymmetric model is always below the one with the symmetric model. The asymmetric model decreases the number of leaves for every topology we have tested, and for all size of acknowledgment traffic.

Since rounds are less constrained in the asymmetric model, i.e. they can contain more links, the solution allows the use of more paths than in the symmetric model $(10 \%$ more in the asymmetric model), leading to a better load distribution in the network (see Table I).

\section{CONCLUSION}

Our results confirm that the couple (MAC layer, topology) has a strong impact on the wireless mesh network capacity. In particular, removing the acknowledgments from the MAC layer and adding them into the transport layer allows to increase the network capacity thanks to an interference limitation. This growth is greater than $20 \%$ for regular gridlike topologies and gateway placement.

Acknowledgments at the transport layer gives also a better load distribution in the network. The amount of traffic flow to forward is balanced between the mesh routers, leading to the use of more paths in the asymmetric interference model.

However, the move of the MAC acknowledgments could induce important delays in the packet delivery in case of transmission failures. This delay would seriously degrade the

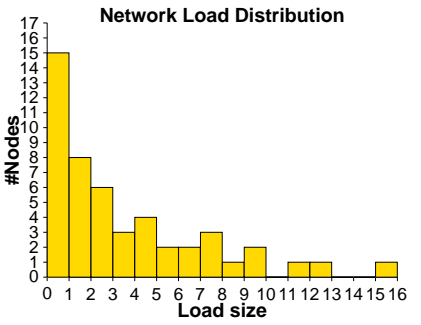

(a) Asymmetric model $($ mean load $=3.43)$.

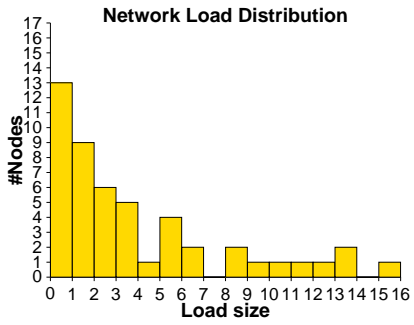

(b) Symmetric model (mean load $=3.49$ ).
Fig. 6. Network load distribution in a random network of 50 nodes.

QoS offered to the clients. A study of the effects of the acknowledgment traffic on the delay is a topic for further research. We would like to do this analysis through simulations using the WSNet simulator [15]. Optimizing the transmission schedule during a time period, as well as evaluating the size of the maximum queue in each mesh router are also interesting prospects we want to go into.

\section{ACKNOWLEDMENT}

C. Molle PhD is funded by DGA, France. This work has been partially supported by ARC CARMA and European project IST/FET AEOLUS.

\section{REFERENCES}

[1] I. Akildiz, X. Wang, and W. Wang, "Wireless mesh networks : a survey," Elsevier Journal of Comupter Networks, vol. 47, pp. 445-487, 2005.

[2] P. Gupta and P. Kumar, "The capacity of wireless networks," IEEE Transactions on Information Theory, vol. 46, no. 2, pp. 388-404, 2000.

[3] J. Jun and M. Sichitiu, "The nominal capacity of wireless mesh networks," IEEE Wireless Communications, vol. 10, no. 5, pp. 8-14, 2003.

[4] P. Du, W. Jia, L. Huang, and W. Lu, "Centralized scheduling and channel assignment in multi-channel single-transceiver wimax mesh network," in IEEE WCNC, 2007, pp. 1736-1741.

[5] P. Djukic and S. Valaee, "Performance comparison of 802.16 centralized scheduling algorithms," Submitted to ACM/Springer Mobile Networks and Applications, 2007.

[6] M. Kodialam and T. Nandagopal, "On the capacity region of multi-radio multi-channel wireless mesh networks," in First IEEE WiMesh, 2005.

[7] K. Jain, J. Padhye, V. Padhamanabhan, and L. Qiu, "Impact of interference on multi-hop wireless network performance," in ACM MobiCom, 2003, pp. 66-80.

[8] R. Klasing, N. Morales, and S. Perennes, "On the complexity of bandwidth allocation in radio networks with steady traffic demands," Theoretical Computer Science, 2008.

[9] C. Molle, F. Peix, and H. Rivano, "An optimization framework for the joint routing and scheduling in wireless mesh networks," in IEEE PIMRC, 2008.

[10] M. E. Lubbecke and J. Desrosiers, "Selected topics in column generation," Operations Research, vol. 53, no. 6, pp. 1007-1023, 2005.

[11] J. Zhang, H. Wu, Q. Zhang, and B. Li, "Joint routing and scheduling in multi-radio multi-channel multi-hop wireless networks," in IEEE BROADNETS, 2005, pp. 678-687.

[12] M. Johansson and L. Xiao, "Cross-layer optimization of wireless networks using nonlinear column generation," IEEE Transactions on Wireless Communications, vol. 5, no. 2, pp. 435-446, 2006.

[13] M. Grotschel, L. Lovasz, and A. Schrijver, "The ellipsoid method and its consequences in combinatorial optimization," Combinatorica, vol. 1, no. 2, pp. 169-197, Jun. 1981.

[14] "Mascopt (mascotte optmization) project." [Online]. Available: http://www-sop.inria.fr/mascotte/mascopt/

[15] "Wsnet simulator." [Online]. Available: http://wsnet.gforge.inria.fr/ 